\title{
Chimera-like states in an array of coupled-waveguide resonators
}

\author{
M. G. Clere, ${ }^{1}$ M. A. Ferré, ${ }^{1, *}$ S. Coulibaly, ${ }^{2}$ R. G. Rojas, ${ }^{3}$ and M. Tlidi ${ }^{4}$ \\ ${ }^{1}$ Departamento de Física, FCFM, Universidad de Chile, Casilla 487-3, Santiago, Chile \\ 2Université de Lille, CNRS, UMR 8523-PhLAM-Physique des Lasers Atomes et Molécules, F-59000 Lille, France \\ ${ }^{3}$ Instituto de Física, Pontificia Universidad Católica de Valparaíso, Casilla 4059, Valparaíso, Chile \\ ${ }^{4}$ Faculté des Sciences, Université Libre de Bruxelles (U.L.B.), CP 231, Campus Plaine, B-1050 Bruxelles, Belgium \\ *Corresponding author: michel.ferre.diaz@gmail.com
}

Received 18 April 2017; revised 26 June 2017; accepted 29 June 2017; posted 30 June 2017 (Doc. ID 292616); published 19 July 2017

\begin{abstract}
We consider coupled-waveguide resonators subject to optical injection. The dynamics of this simple device are described by the discrete Lugiato-Lefever equation. We show that chimera-like states can be stabilized, thanks to the discrete nature of the coupled-waveguide resonators. Such chaotic localized structures are unstable in the continuous Lugiato-Lefever model; this is because of dispersive radiation from the tails of localized structures in the form of two counter-propagating fronts between the homogeneous and the complex spatiotemporal state. We characterize the formation of chimera-like states by computing the Lyapunov spectra. We show that localized states have an intermittent spatiotemporal chaotic dynamical nature. These states are generated in a parameter regime characterized by a coexistence between a uniform steady state and a spatiotemporal intermittency state. () 2017 Optical Society of America
\end{abstract}

OCIS codes: (070.5753) Resonators; (190.4370) Nonlinear optics, fibers; (190.3100) Instabilities and chaos; (140.1540) Chaos; (230.7370) Waveguides.

https://doi.org/10.1364/OL.42.002906

The existence of conservative discrete solitons in periodic nonlinear systems such as discrete arrays of coupled waveguides $[1,2]$ or Bose-Einstein condensate [3] has been abundantly discussed and is by now fairly well understood. Most of the theoretical studies have been developed using the discrete nonlinear Schrödinger equation. However, when dielectric mirrors are placed at the input and output facet of waveguide arrays, it is possible to stabilize dissipative discrete solitons, thanks to the balance between losses and injection of light beams. This simple optical device can be seen as interacting microcavities in photonic crystals. The dynamics of this dissipative system cannot be described by the nonlinear Schrödinger equation, but rather by the discrete Lugiato-Lefever equation which admits both stationary and oscillating discrete dissipative solitons often called discrete localized structures [4-6]. Recently, the observation of spatiotemporal localized chaotic states has been reported $[7,8]$. Similar behavior has been reported in a broad-area vertical-cavity surface-emitting-laser with saturable absorption subject to time-delayed optical feedback [9]. In the continuous limit, the Lugiato-Lefever equation exhibits fronts between steady states. These fronts are motionless at a single point often called the Maxwell point [10]. Near this point, one expects to find localized states as the result of front interaction [11-14].

Coupled oscillators under the influence of injection and dissipation of energy exhibit a coexistence between coherent and incoherent behavior, known as chimera states $[15,16]$. More recently, it has been shown that the discrete nature of coupling is a key ingredient in the emergence of chimera-like states [17]. Hence, the existence of chimera states in the discrete LugiatoLefever equation is expected.

In this Letter, we show that the discrete Lugiato-Lefever equation supports chimera-like states in an array of coupledwaveguide resonators. They consist of spatiotemporal chaos embedded in a homogeneous background. They correspond to a coexistence in the same system of coherent and incoherent states. We characterize chimera-like states by computing Lyapunov spectra. Note, however, that in the continuous Lugiato-Lefever model, it is not possible to stabilize chimeralike state because of the radiation emanating from localized state in the form of two counter-propagating fronts between the homogeneous and the complex spatiotemporal states $[18,19]$. Therefore, we attribute the formation of chimera-like states to the discrete nature of interacting microcavities in photonic crystals.

We consider an array of weakly coupled nonlinear waveguides where mirrors at the input and the output facet back-fold the light path, thus forming an array of coupledwaveguide resonators, which is excited by an external driving field $E_{0}$. Figure 1 depicts an array of coupled-waveguide resonators. The resonators are then endowed with a self-focusing Kerr medium. The slowly varying envelope $\psi_{n}$ of the field circulating in the $n$th waveguide resonator can be derived by using the mean-field approach developed in $[4,5]$

$\partial_{T} \psi_{n}=E_{0}-(1+i \Delta) \psi_{n}-i\left|\psi_{n}\right|^{2} \psi_{n}-i C\left(\psi_{n+1}-2 \psi_{n}+\psi_{n-1}\right)$. 
(a)

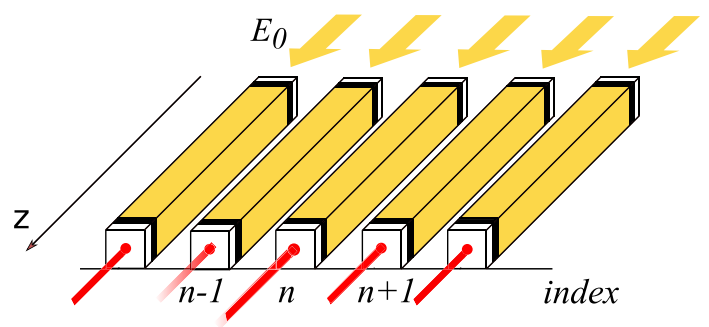

(b) 18
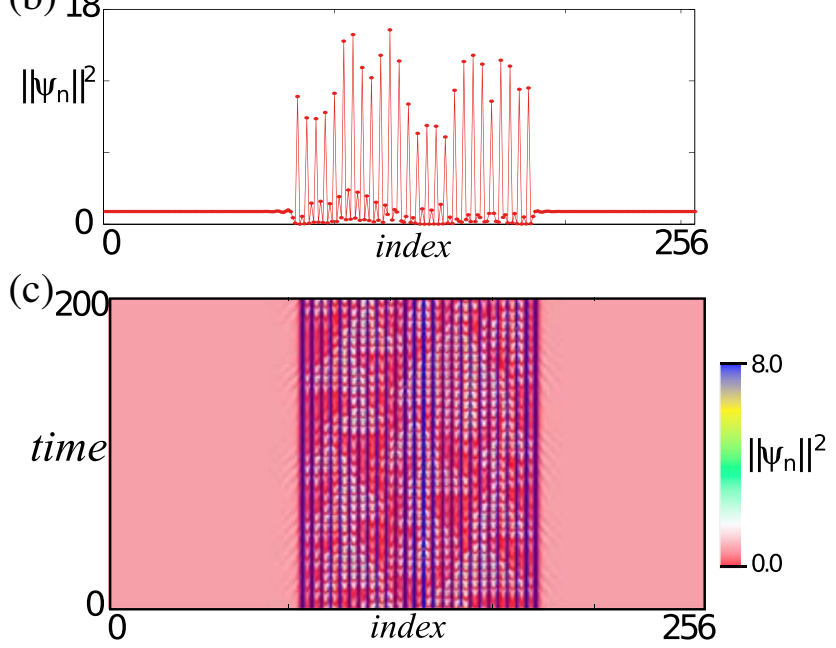

Fig. 1. Chimera-like states in an array of coupled-waveguide resonators obtained by numerical simulations of Eq. (1). (a) Schematic representation of an array of coupled-waveguide resonators driven by an external electrical field intensity $E_{0}^{2}$ with operating frequency $\omega$ close to the resonance cavity frequency $\omega_{0}$. (b) Intensity profile of the electric field amplitude $\psi_{n}(t)$ at a fixed time. (c) Spatiotemporal evolution of the intensity $\left\|\psi_{n}(t)\right\|^{2}$. The parameters are $E_{0}=6.2, \Delta=7.0$, and $C=1.9$.

Here, the evolution time $t=T \tau_{p h}$ is measured in the photon lifetime unit $\tau_{p h} ; \Delta \equiv \omega-\omega_{0}$ accounts for detuning between a resonance frequency $\omega_{0}$ of the cavities and the carrier of the pumping field frequency $\omega$. The parameter $C$ denotes the nearest-neighbor coupling constant. The parameter $E_{0}$ can be considered as a positive and real in order to fix the origin of the phase.

The continuous counterpart of the discrete model Eq. (1), $C \rightarrow \infty$, is known as the Lugiato-Lefever equation [20] or the driven damped nonlinear Schrödinger equation [21] in the optical and nonlinear coupled oscillators frameworks, respectively. In the continuous limit, theoretical $[13,19,20]$ and experimental [22] investigations have revealed a coexistence between the flat solution and the spatiotemporal chaotic state. Hence, one expects to observe complex spatiotemporal dynamical behavior in the discrete model Eq. (1). Figure 1 shows the profile and spatiotemporal evolution of a complex localized state obtained from the numerical simulation of discrete Eq. (1). All numerical simulations were conducted using finite difference code with the Runge-Kutta fourth-order algorithm and Neumann boundary conditions that are compatible with a finite array of coupled waveguides. These localized structures correspond to a coexistence of coherent and incoherent states. In the context of coupled oscillators, this type of localized state is usually termed a chimera state [15-17]. Considering a homogeneous initial condition with a small (large) intensity, the system exhibits as an equilibrium solution a uniform state (complex spatiotemporal state). For an inhomogeneous perturbation of the homogeneous state, it is possible to generate a chimera-like state. Figure 2 displays different chimera states obtained by numerical simulations for the same parameter values, but with different initial conditions. From this figure, we can see that the larger the incoherent domain (size of the chimera), the broader the Lyapunov spectrum indicating increasing complexity in the spatiotemporal evolution of the system.

As a matter of fact, in the continuous limit, these intrigued localized states are not observed. That is, when increasing the nearest neighbor coupling constant $C$, depending on the other parameters, the complex localized structure shrinks or spreads so that the system exhibits only one type of extended state as an equilibrium, being either a homogeneous state or a spatiotemporal complex state. Therefore, chimera-like states are a consequence of the discrete nature of the system under study [17]. The complex dynamical behavior can be characterized by the numerical calculation of Lyapunov exponents. These provide information about permanent dynamic with sensitivity to initial conditions [23]. When the largest Lyapunov exponent is positive, the system develops chaotic dynamics, but not necessarily a spatiotemporal chaos [23]. To distinguish between chaos and spatiotemporal chaos, it is necessary to determine the Lyapunov spectrum. This spectrum is composed of a set

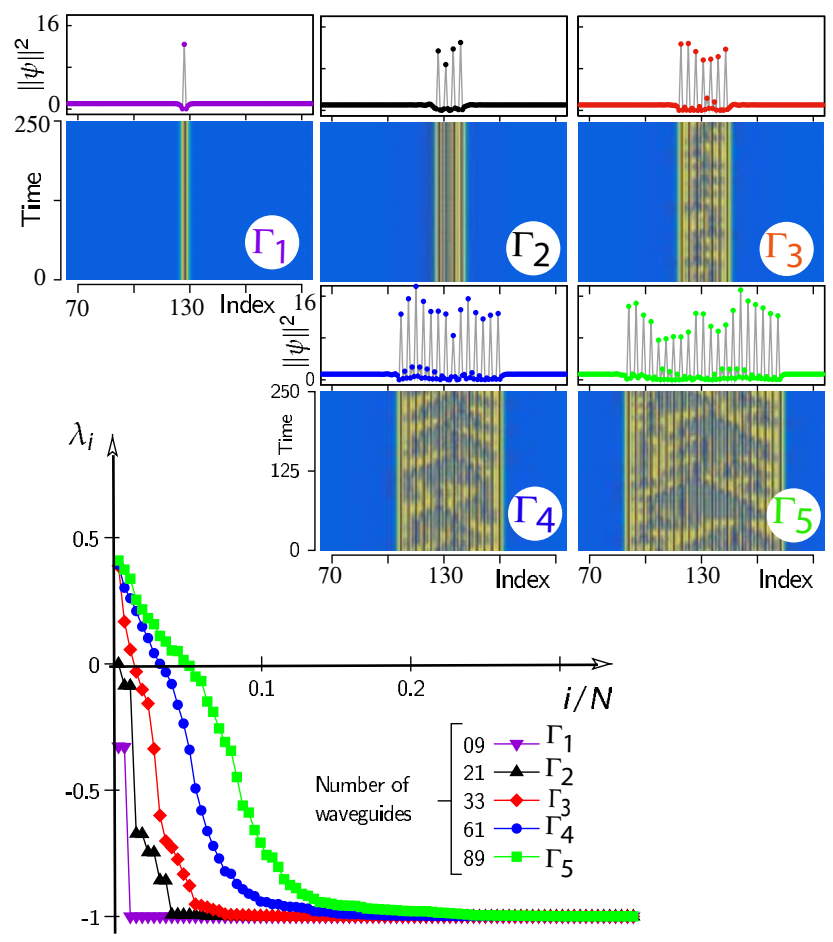

Fig. 2. Lyapunov spectra of different localized states obtained from numerical simulations of the model Eq. (1). The Lyapunov exponents are denoted by $\left\{\lambda_{i}\right\}$, where $i$ labels the exponents $(i=1, \ldots, N), N$ accounts for the number of waveguides, and $\lambda_{p} \leq \lambda_{q}(p \geq q) . \Gamma_{1}, \Gamma_{2}$, $\Gamma_{3}, \Gamma_{4}$, and $\Gamma_{5}$ stand for localized structures with different widths. $\Gamma_{1}$ is stationary, and $\Gamma_{2}$ is the oscillatory localized state. $\Gamma_{3,4,5}$ are chimera states. The insets describe the profile and the spatiotemporal evolution of the $\Gamma_{l}$ localized structure $(l=\{1,2,3,4,5\})$. The parameters are $E_{0}=6.2, \Delta=7.0$, and $C=1.9$. 
of exponents. Spatiotemporal chaos has a Lyapunov spectrum with a continuous set of positive values. In contrast, chaos possesses a Lyapunov spectrum with a discrete set of positive values. Another feature that distinguishes these dynamic behaviors is when one increases the system size; for spatiotemporal chaos, the number of positive Lyapunov exponents grows (extensive property) and, for chaos, positive Lyapunov exponents remain constant (intensive property). To understand the nature of the predicted chimera-like states from the point of view of the dynamical system theory, we have computed numerically the Lyapunov exponents of localized states, using the strategy proposed in $[24,25]$. Figure 2 shows Lyapunov spectra of different chimera-like states. Therefore, we infer that complex localized structures are of spatiotemporal chaotic nature. Namely, the dynamical behaviors presented in Figs. 1 and 2 correspond to localized spatiotemporal chaos. Figure 2 shows that chimeralike states with a small size have a smaller number of positive Lyapunov exponents. Hence, the dynamical behavior of a larger chimera is more complex. Indeed, the dimension of the associated strange-attractor is larger [26]. This type of behavior for chimera states of different sizes has been reported [17]. The largest Lyapunov exponents for the different chimera sizes are similar, as shown in Fig. 2. Indeed, it is a manifestation that the most unstable mode for the different chimera states is similar.

Another feature that can be seen from the spatiotemporal diagrams in Figs. 1 and 2 is the spatiotemporal intermittent nature of chimera states. The intermittent type of dynamics is characterized by orderless alternations between a regular and an irregular behavior in their spatiotemporal evolution. Likewise, the spatiotemporal diagram exhibits a Sierpinski carpet type structure (see Fig. 3). This complex dynamic is usually related to defect turbulence of an oscillatory dynamics [27], which is consistent with our numerical observations. Indeed, smaller localized states are stationary or oscillatory, preventing the emergence of defects in the localized state. In contrary, localized states with larger size favor the emergence of defects leading to more complex spatiotemporal dynamical behavior. Figure 3(a) depicts the coexistence within the same system of three localized states: stationary, self-pulsating, and chimera-like state. The bifurcation diagram of localized states as a function of the waveguide number is presented in Fig. 3(b). Notice that there is a minimum width below which chimera states cannot exist.

In the continuous system, front interaction is attractive, and then spatiotemporal localized states are unstable. However, when considering a moderate nearest-neighbor coupling constant $[C \sim O(1)]$, it is possible to stabilize spatiotemporal localized states in the form of a chimera-like state. It has been shown that local coupling in dissipative systems causes a propagation failure or pinning of fronts, in a large region of parameters known as a pinning range [28,29]. Namely, fronts connecting different states become motionless in a large parameter space. This phenomenon, a result of discreteness, induces an effective potential on the front dynamics, which is characterized by the overlap of the attractive interaction of fronts and the PeierlsNabarro potential [30]. Hence, the local coupling prevents the irregular state to invade the coherent one. Indeed, the PeierlsNabarro potential induced by discreteness generates a family of stable localized spatiotemporal chaotic states with different sizes.
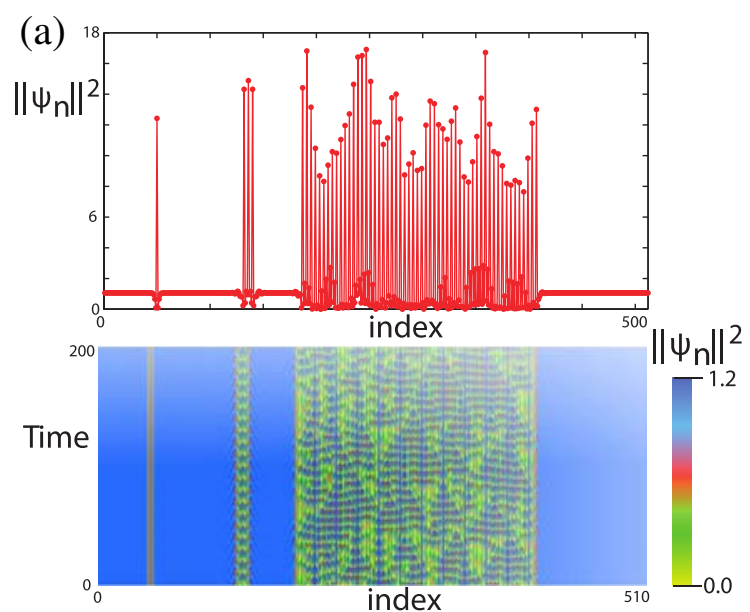

(b)

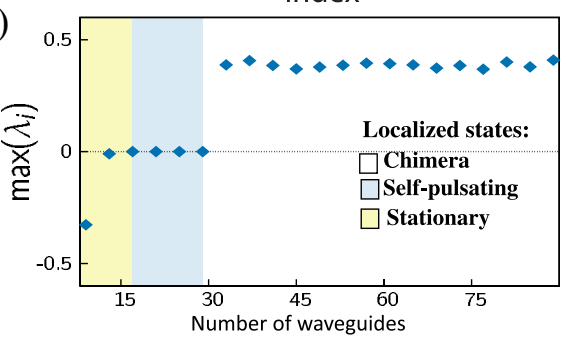

Fig. 3. (a) Coexistence of three localized states: a stationary, an oscillatory, and a spatiotemporal intermittent localized state obtained from numerical simulations of model Eq. (1) by $E_{0}=6.2, \Delta=7.0$, and $C=1.9$. The top panel shows the instantaneous profile of $\left\|\psi_{n}(t)\right\|^{2}$, and the bottom panel depicts the spatiotemporal evolution of the amplitude of a slowly varying envelope. (b) Bifurcation diagram of localized states as a function of the number of the waveguides, where $\max \left(\lambda_{i}\right)$ accounts for the largest Lyapunov exponent.

Therefore, these chimera states exhibited by an array of coupled-waveguide resonators are the result of local coupling.

Quantitatively, the spatiotemporal intermittency is characterized by a power law decay in the spectrum [27]. Figure 4 displays the temporal average power spectrum of chimera-like states computed from numerical simulations of the model

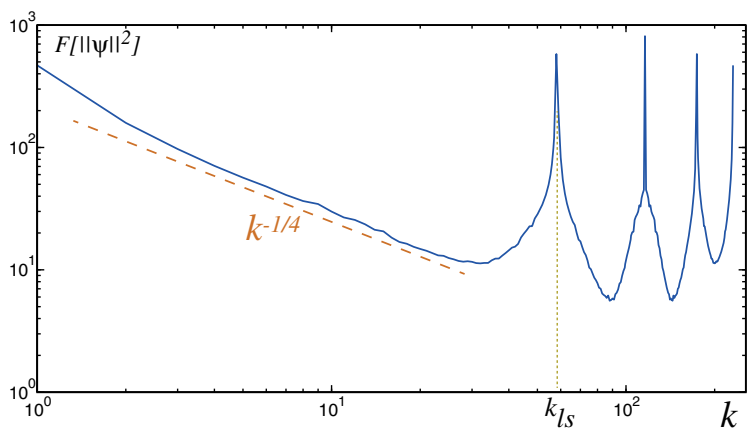

Fig. 4. Log-log plot of average power spectrum of chimera-like state amplitude $F\left[\|\psi\|^{2}\right]$ as a function of the wavenumber $k$, obtained from numerical simulations of model Eq. (1) with $E_{0}=6.2, \Delta=6.0$, and $C=1.9$, after considering 16,000 temporary steps. The dashed line is a $k^{-1 / 4}$ fit slope. $k_{l s}$ accounts for the typical wavelength of a single peak. For small wave numbers, the system exhibits a power law that is a manifestation of the spatiotemporal intermittency. 
Eq. (1). One can infer that for small wave numbers, the system exhibits a power law characterized by a $1 / 4$ decreasing exponent (see the dashed curve in Fig. 4). This tendency persists until the system reaches the typical wavelength $k_{l s}$ corresponding to the distance between the oscillating pulses forming the chimera-like state. This behavior contrasts strongly with the continuous limit where the power spectrum exhibits a triangular shape [31].

In conclusion, we have shown the existence of chimera-like states in the discrete Lugiato-Lefever model describing an array of coupled-waveguide resonators. These states consist of a localized spatiotemporal chaos embedded in a homogeneous background. We have established Lyapunov spectra to show that these localized complex states exhibit a spatiotemporal chaos. In turn, based on the spatiotemporal evolution, we have shown that complex dynamics are of a spatiotemporal intermittency type. We have analyzed the dynamics of an array of coupledwaveguide resonators, with a coupling parameter of order 1 . However, at the weak coupling limit, one expects to find a rich spatiotemporal dynamics. A study in this direction is in progress. Experimentally, it is difficult to get cavities with identical properties. Numerically, we have conducted simulations with inhomogeneous parameters and an additive noise. We observe that the chimera states are robust.

Funding. Comisión Nacional de Investigación Científica y Tecnológica (CONICYT) (PII20150011); Belgian Science Policy Office (IAPP7-35); Agence Nationale de la Recherche (ANR) (OptiRoc N12-BS04-0011); Fonds National de la Recherche Scientifique (Belgium); Evaluation-orientation de la Coopération Scientifique (ECOS) (C15E06).

Acknowledgment. M. Clerc and M. Ferré are grateful for the financial support of CONICYT-USA. S. Coulibaly and M. Tlidi thank the Interuniversity Attraction Poles program of the Belgian Science Policy Office, the French Project ANR Blanc and the "Laboratoire d'Excellence: Centre Européen pour les Mathématiques, la Physique et leurs Interactions" CEMPI. M. Tlidi received support from the Fonds National de la Recherche Scientifique (Belgium). R. Rojas and S. Couibaly are grateful for the financial support of ECOS-CONICYT.

\section{REFERENCES}

1. D. N. Christodoulides and R. I. Joseph, Opt. Lett. 13, 794 (1988).

2. J. W. Fleischer, M. Segev, N. K. Efremidis, and D. N. Christodoulides, Nature 422, 147 (2003).

3. A. Trombettoni and A. Smerzi, Phys. Rev. Lett. 86, 2353 (2001).

4. U. Peschel, O. Egorov, and F. Lederer, Opt. Lett. 29, 1909 (2004).

5. O. Egorov, U. Peschel, and F. Lederer, Phys. Rev. E 71, 056612 (2005).

6. O. A. Egorov and F. Lederer, Opt. Lett. 38, 1010 (2013).

7. N. Verschueren, U. Bortolozzo, M. G. Clerc, and S. Residori, Phys. Rev. Lett. 110, 104101 (2013).

8. N. Verschueren, U. Bortolozzo, M. G. Clerc, and S. Residori, Philos. Trans. R. Soc. London A 372, 20140011 (2014).

9. K. Panajotov and M. Tlidi, Opt. Lett. 39, 4739 (2014).

10. S. Coen, M. Tlidi, Ph. Emplit, and M. Haelterman, Phys. Rev. Lett. 83 , 2328 (1999).

11. P. Coullet, Int. J. Bifurcation Chaos 12, 2445 (2002).

12. M. G. Clerc and C. Falcon, Physica A 356, 48 (2005).

13. P. Parra-Rivas, D. Gomila, M. A. Matias, S. Coen, and L. Gelens, Phys. Rev. A 89, 043813 (2014).

14. P. Parra-Rivas, E. Knobloch, D. Gomila, and L. Gelens, Phys. Rev. A 93, 063839 (2016).

15. D. M. Abrams and S. H. Strogatz, Phys. Rev. Lett. 93, 174102 (2004).

16. Y. Kuramoto and D. Battogtokh, Nonlinear Phenom. Complex Syst. 5, 380 (2002).

17. M. G. Clerc, S. Coulibaly, M. Ferré, M. A. García-Ñustes, and R. G. Rojas, Phys. Rev. E 93, 052204 (2016).

18. F. Leo, L. Gelens, P. Emplit, M. Haelterman, and S. Coen, Opt. Express 21, 9180 (2013)

19. Z. Liu, M. Ouali, S. Coulibaly, M. G. Clerc, M. Taki, and M. Tlidi, Opt. Lett. 42, 1063 (2017)

20. L. A. Lugiato and R. Lefever, Phys. Rev. Lett. 58, 2209 (1987).

21. K. Nozaki and N. Bekki, Phys. Lett. A 102, 383 (1984).

22. M. Anderson, F. Leo, S. Coen, M. Erkintalo, and S. G. Murdoch, Optica 3, 1071 (2016).

23. A. Pikovsky and A. Politi, Lyapunov Exponents: A Tool to Explore Complex Dynamics (Cambridge University, 2016).

24. F. Christianen and H. H. Rough, Nonlinearity 10, 1063 (1997).

25. T. J. Bridges and S. Reich, Physica D 156, 219 (2001).

26. E. Ott, Chaos in Dynamical Systems, 2nd ed. (Cambridge University, 2002).

27. H. Chate, Nonlinearity 7, 185 (1994).

28. G. Fath, Physica D 116, 176 (1998).

29. M. G. Clerc, R. G. Elias, and R. G. Rojas, Philos. Trans. R. Soc. A 369, 412 (2011).

30. M. O. Braun and Y. Kivshar, The Frenkel-Kontorova Model: Concepts, Methods, and Applications (Springer, 2013).

31. N. Akhmediev, A. Ankiewicz, J. M. Soto-Crespo, and J. M. Dudley, Phys. Lett. A 375, 775 (2011). 